\title{
A Novel Predictive Marker for In-Hospital Mortality in Acute Cerebral Infarction: Low- Density Lipoprotein Cholesterol to Lymphocyte Ratio
}

Muzaffer Güneş ${ }^{1}$, Hüseyin Büyükgöl ${ }^{2}$

1. Neurology, Aksaray University Training and Research Hospital, Aksaray, TUR 2. Neurology, KTO Karatay University, Konya, TUR

Corresponding author: Muzaffer Güneş, drmuzaffergunes@gmail.com

\section{Abstract \\ Objectives}

Neutrophil to eosinophil ratio, neutrophil to lymphocyte ratio, C-reactive protein to albumin ratio, and red blood cell distribution width (RDW) have been studied in patients with acute cerebral infarction (ACI). However, the low-density lipoprotein cholesterol (LDL-C) to lymphocyte ratio has never been studied. Hence, our objective was to study the LDL-C to lymphocyte ratio with regard to in-hospital mortality rates of patients with ACI.

\section{Materials and methods}

We retrospectively examined our patients diagnosed with ACI between January 2015 and December 2018. The patients' clinical data and imaging and laboratory results during the acute period were retrieved from our database and saved for statistical analysis. The receiver operating characteristic (ROC) curve analysis was performed to evaluate the predictive value of the variables and to calculate the cut-off values.

\section{Results}

A total of 172 patients with ACI, including 43 patients who died and 129 patients who survived in the hospital, were included in the study. The median age of the patients who died was significantly higher than that of those who survived (p: $<0.001$ ). Median triglyceride level, LDL-C to lymphocyte ratio, and RDW-SD values were significantly higher in patients who died $(\mathrm{p}=0.017, \mathrm{p}:<0.001$, and $\mathrm{p}=0.003$, respectively). Areas under the ROC curve were found to be as follows: LDL-C to lymphocyte ratio: 0.774 (95\% CI: 0.697-0.851), RDW-SD: 0.562 (95\% CI: 0.456-0.669), and triglyceride level: 0.621 (95\% CI: 0.531-0.732). The cut-off value of the LDL-C to lymphocyte ratio was 59.71 (sensitivity: $79.1 \%$; specificity: $58.1 \%$ ).

Received 08/07/2020

Review began 08/11/2020 Review ended 08/14/2020 Published 08/24/2020

\section{() Copyright 2020}

Güneș et al. This is an open access article distributed under the terms of the Creative Commons Attribution License CC-BY 4.0., which permits unrestricted use, distribution, and reproduction in any medium, provided the original author and source are credited.

\section{Conclusions}

The LDL-C to lymphocyte ratio can be used as a marker to predict in-hospital mortality in patients with ACI. We recommend further studies to verify our findings.

\author{
Categories: Neurology \\ Keywords: acute cerebral infarction, low density lipoprotein-cholesterol, lymphocyte, stroke, high density
} lipoprotein-cholesterol, red blood cell distribution width

\section{Introduction}

Stroke is one of the major causes of disability and death in adults [1]. Acute cerebral infarction (ACI) accounts for approximately $80 \%$ of all strokes [2]. The mortality rates for ACI with large artery occlusion is still high [3]. Patient age and the severity of the stroke are known as the key determinants of poor prognosis [4]. Knowledge of prognostic factors can contribute to the management of stroke by clinicians. Therefore, investigators have been focusing on new and effective prognostic factors relating to ACI. In recent studies, hematological parameters, such as neutrophil to lymphocyte ratio [5], monocyte to high-density lipoprotein cholesterol (HDL-C) ratio [6], C-reactive protein to albumin ratio [7], red blood cell distribution width (RDW) [8], and the neutrophil to eosinophil ratio [9], were found to be associated with the prognosis of ACI.

The increase in low-density lipoprotein cholesterol (LDL-C) levels is known to be a risk factor for ischemic stroke [10,11]. Moreover, high LDL-C levels are associated with clinical deterioration in patients with ACI [12]. In recent years, studies have found that during the acute period, blood lymphocyte counts of patients with ACI who died were lower than those of patients who survived [8]. Therefore, the LDL-C to lymphocyte ratio can be a better and different expression of the increased LDL-C level and reduced lymphocyte count in the blood. In light of this information, the LDL-C to lymphocyte ratio could predict the in-hospital mortality 
of patients with ACI. To the best of our knowledge, the LDL-C to lymphocyte ratio in ACI has never been investigated. Hence, in this study, we aimed to investigate the LDL-C to lymphocyte ratio in ACI.

\section{Materials And Methods}

Patients who were treated for the diagnosis of ACI and underwent subsequent follow-ups at the Neurology Department of Aksaray University Training and Research Hospital between January 2015 and December 2018 were retrospectively examined. Patients who presented to our emergency room within the first 24 hours from the onset of symptoms were regarded as ACI, and the study was conducted with these patients. The subjects included in the study were divided into two groups. The first group comprised those who died from ACI that developed due to a detected cerebrovascular occlusion, while the second group comprised those who did not die from ACI (control group). Only those patients who were older than 18 years were included in the study. Subjects whose symptoms started more than 24 hours before the emergency room visit; those who were taking immunosuppressive drugs; those with an indication for mechanical thrombectomy and therefore referred; those with hematological disorders, liver and kidney disease, hemorrhagic infarction, electrolyte imbalance; and those who were under 18 years of age as well as those with missing data were excluded from the study. The study was conducted with laboratory data of patients obtained within the first 24 hours following the onset of symptoms.

Initially, medical history was obtained from patients who were brought to our hospital with a preliminary diagnosis of stroke or from their relatives. Subsequently, patients' vital signs (body temperature, blood pressure, oxygen saturation, pulse, etc.), as well as finger stick blood glucose levels, were measured. Neurological evaluation was performed, while blood samples were taken for hemogram, international normalized ratio, activated partial thromboplastin time, and biochemical evaluation. For parenchymal imaging of the brain, CT of the brain or MRI of the brain was performed. In addition, computed tomography angiography of the brain or magnetic resonance angiography of the brain was performed for vascular imaging of the brain. Consequently, the clogged arteries of the patients were determined and the treatment to be performed was quickly planned accordingly. Patients with mechanical thrombectomy indications were referred. Other patients received treatment according to the most recent stroke guidelines [13]. Of these, 14 patients who met the intravenous (IV) thrombolytic therapy criteria were given IV thrombolytic therapy.

Peripheral venous blood samples were taken from all patients and centrifuged, and then, blood cell count analyses were performed using an autoanalyzer (Sysmex XN-1000 Hematology Analyzer; Sysmex Corporation, Kobe, Japan) in the hematology laboratory of our institution [9]. LDL-C to lymphocyte ratio was calculated by simply dividing the LDL-C amount by the lymphocyte count.

Information on clinical and laboratory findings, results of the parenchymal and vascular imaging of the brain, risk factors, and other demographic characteristics of the patients registered in our database were retrieved and saved for statistical analysis. The study was approved by the ethics committee of our institution and was carried out in accordance with the Declaration of Helsinki.

\section{Statistical analysis}

Results were presented as mean \pm standard deviation for normally distributed data and as median (minimum-maximum) for abnormally distributed data. To investigate the distribution pattern of the data, the Kolmogorov-Smirnov normality test was used. Only red blood cell (RBC) data distributed normally, and hence were compared using Student's independent samples T-test; other blood test parameters did not distribute normally, and hence were compared using the Mann-Whitney U test. To compare the gender distribution of the groups, we used the chi-square test. To assess the predictive value of variables, the receiver operating characteristics (ROC) curve analysis test was used [9,14]. If the area under the ROC curve was 0.5 , the model did not discriminate; if it was $0.5-0.7$, the model had poor to fair discrimination; if it was 0.7-0.8, the model had acceptable discrimination; if it was was between 0.8-0.9, the model had excellent discrimination; a value of 0.9-1.0 was a very rare outcome. For statistical analysis of all data, we used SPSS Statistics version 23.0 software for Windows (IBM, Armonk, NY). A p-value of less than 0.05 was considered statistically significant.

\section{Results}

A total of 172 patients with ACI were eligible for the study. The group without hospital mortality (survivors) consisted of 129 patients [67 males and 62 females, median age: 69 years (range: 22-93)] and the group with hospital mortality (non-survivors) consisted of 43 patients [15 males and 28 females, median age: 78 years (range: 58-99)]. The groups were gender-matched $(\mathrm{p}=0.052)$, but the median age of the patients with hospital mortality was significantly higher compared to those without mortality (p: <0.001).

The comparison of blood parameters between the groups (survivors and non-survivors) is presented in Table 1. According to the Student's T-test, the RBC value did not significantly differ between the groups $(\mathrm{p}=0.109)$. The Mann-Whitney U test revealed that the median total cholesterol, neutrophil to HDL-C ratio, monocyte to HDL-C ratio, white blood cell (WBC), RDW-CV, mean corpuscular volume and platelet values did not significantly differ between the patients with and without hospital mortality $(p=0.116, p=0.230, p=0.690$, 


\section{Cureus}

$\mathrm{p}=0.844, \mathrm{p}=0.222, \mathrm{p}=0.353$, and $\mathrm{p}=0.513$, respectively). However, the median triglyceride, $\mathrm{LDL}-\mathrm{C}$ to lymphocyte ratio, and RDW-SD values were significantly higher in patients with in-hospital mortality, compared with the survivors $(\mathrm{p}=0.017, \mathrm{p}:<0.001$ and $\mathrm{p}=0.003$, respectively).

\begin{tabular}{|c|c|c|c|}
\hline Parameters & $\begin{array}{l}\text { Survivors (those without in-hospital } \\
\text { mortality) }\end{array}$ & $\begin{array}{l}\text { Non-survivors (those with in-hospital } \\
\text { mortality) }\end{array}$ & P-value \\
\hline RBC $\left(10^{12} / \mathrm{L}\right)$ & $4.86 \pm 0.67$ & $4.67 \pm 0.64$ & 0.109 \\
\hline $\begin{array}{l}\text { Total cholesterol } \\
\text { (mg/dL) }\end{array}$ & $178(111-327)$ & $195(131-382)$ & 0.116 \\
\hline Triglyceride (mg/dL) & $128(56-366)$ & $160(70-322)$ & 0.017 \\
\hline LDL/lymphocyte & 54.48 (12.26-156.06) & 89.19 (41.32-202.39) & $<0.001$ \\
\hline Neutrophil/HDL & $0.146(0.047-0.57)$ & $0.126(0.057-0.56)$ & 0.230 \\
\hline Monocyte/HDL & $0.015(0.001-0.087)$ & $0.013(0.003-0.047)$ & 0.690 \\
\hline WBC $\left(10^{9} / \mathrm{L}\right)$ & $8.7(4.13-19.03)$ & $8.71(5.26-22.91)$ & 0.844 \\
\hline RDW-CV (\%) & 13.3 (10.9-26) & 13.8 (10.3-23.5) & 0.222 \\
\hline RDW-SD (fL) & $43.4(27.9-70)$ & $45.1(38.1-57.6)$ & 0.003 \\
\hline MCV (fL) & $87.3(60.3-99.6)$ & $89.6(68-102.6)$ & 0.353 \\
\hline Platelet $\left(10^{9} / \mathrm{L}\right)$ & $227(96-518)$ & $219(111-408)$ & 0.513 \\
\hline
\end{tabular}

\section{TABLE 1: Comparison of blood parameters between survivor and non-survivor groups}

RBC: red blood cell; LDL: low-density lipoprotein; HDL: high-density lipoprotein; WBC: white blood cell; RDW: red blood cell distribution width; MCV: mean corpuscular volume

Figure 1 shows the ROC curve representing the predictive value of LDL-C to lymphocyte ratio, RDW-SD, and triglyceride, for mortality. The areas under curve were as follows: LDL-C to lymphocyte ratio: 0.774 (95\% CI: 0.697-0.851), RDW-SD: 0.562 (95\% CI: 0.456-0.669), and triglyceride: 0.621 (95\% CI: 0.531-0.732). The cutoff value of LDL-C to lymphocyte ratio indicating mortality in patients with ACI was found to be 59.71 (sensitivity: $79.1 \%$; specificity: $58.1 \%$ ). 


\section{Cureus}

ROC Curve

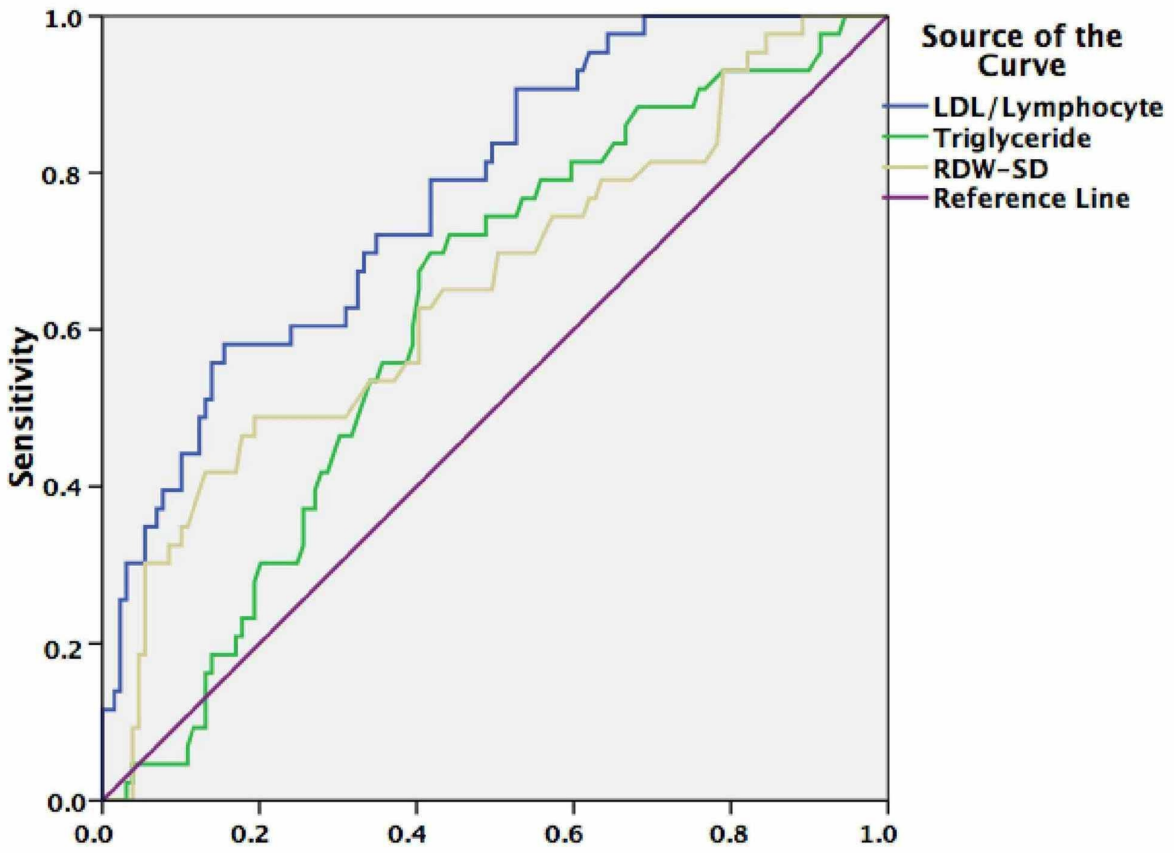

1 - Specificity

FIGURE 1: Receiver operating characteristic (ROC) curve representing the predictive value of the low-density lipoprotein cholesterol (LDL-C) to lymphocyte ratio, red blood cell distribution width-standard deviation (RDW-SD), and triglyceride level for mortality

\section{Discussion}

This study showed that an early high LDL-C to lymphocyte ratio is an independent predictor of in-hospital mortality in patients with ACI. In the present study, we used the laboratory data within the first 24 hours following the onset of the patients' symptoms to determine whether they are early predictors of mortality. To the best of our knowledge, this is the first study investigating the LDL-C to lymphocyte ratio in ACI.

Although it has been revealed in recent years that some inflammatory cells, such as neutrophils [15] and pro-inflammatory cytokines [16], play an important role in secondary brain damage in ACI, the pathophysiological mechanisms of secondary brain damage are still not clear yet. Therefore, this issue remains a focus for neuroscientists.

Secondary brain damage in ACI occurs with a chain of pathophysiological events involving a number of inflammatory cells. Neutrophils have particularly played an important role in this damage. Cell necrosis begins soon after the stroke, followed by increased reactive oxygen species in this area, and activation of microglia and astrocytes begins with the chemokines and cytokines released. Overactivation of microglia and astrocytes leads to the release of chemokines, pro-inflammatory cytokines, and free oxygen species $[16,17]$. They, consequently, cause the passage of neutrophils produced in peripheral blood to the ischemic brain region approximately four to six hours after ACI [16,17]. Increased neutrophils in and around the ischemic region of the brain contribute to oversecretion of pro-inflammatory cytokines, overproduction of reactive oxygen species, overregulation of matrix metalloproteinase-9, and macrophage activation [16-18]. The endpoint reached with this chain of pathophysiological events is secondary brain damage caused by the disruption of the blood-brain barrier, neuronal death, increased edema, and enlargement of the ischemic area [16-18]. In ACI, the neutrophil count increases, while the lymphocyte count decreases [5,19]. Although a correlation exists between high LDL-C levels and clinical deterioration [12] and long-term mortality [20] in patients with ACI, its relationship with mortality is not clear [21]. In light of this information, we believe that the LDL-C to lymphocyte ratio may represent a better and different expression of the increase in the amount of LDL-C and the decrease in lymphocyte count in the blood. The LDL-C to lymphocyte ratio is important as it has a better predictive value than RDW in terms of its potential to predict the mortality of ACI in the present study. Finally, due to these significant results obtained in the present study, we believe that the LDL-C to lymphocyte ratio can be used as a marker that can predict in-hospital mortality in patients with ACI. We believe that further studies conducted on this subject in the future can verify this. In 
the present study, we found that in addition to the LDL-C to lymphocyte ratio, RDW-SD predicts mortality in patients with ACI. This result is consistent with that of previous studies $[5,8]$.

Although this study was conducted to determine whether the early LDL-C to lymphocyte ratio has a predictive value in the in-hospital mortality of patients with ACI and to emphasize the importance of this in ACI, it also has some limitations. They include the relatively low number of patients we included in the study, the absence of comparison of the laboratory parameters between acute and subacute periods of the patients, and the single-center and retrospective nature of the study.

\section{Conclusions}

Based on our findings, an early high LDL-C-to-lymphocyte ratio can predict the in-hospital mortality of patients with ACI. It may even have a better predictive value than RDW-SD. Further studies on LDL-C to lymphocyte ratio with a higher number of patients are needed to implement it as a new predictive marker in ACI.

\section{Additional Information \\ Disclosures}

Human subjects: Consent was obtained by all participants in this study. Aksaray University Human Research Ethics Committee issued approval 2020/06-39. This study was approved by the Aksaray University Human Research Ethics Committee. Animal subjects: All authors have confirmed that this study did not involve animal subjects or tissue. Conflicts of interest: In compliance with the ICMJE uniform disclosure form, all authors declare the following: Payment/services info: All authors have declared that no financial support was received from any organization for the submitted work. Financial relationships: All authors have declared that they have no financial relationships at present or within the previous three years with any organizations that might have an interest in the submitted work. Other relationships: All authors have declared that there are no other relationships or activities that could appear to have influenced the submitted work.

\section{Acknowledgements}

The authors would like to thank Associate Professor Dr. Ö. Hızlı and Assistant Professor Dr. P. Güneş for their assistance with the statistical analysis. Also, the authors thank Enago https://www.enago.com.tr/ceviri/ - for their help in manuscript translation and editing.

\section{References}

1. Lopez AD, Mathers CD, Ezzati M, Jamison DT, Murray CJ: Global and regional burden of disease and risk factors, 2001: systematic analysis of population health data. Lancet. 2006, 367:1747-1757. 10.1016/S01406736(06)68770-9

2. Adams HP Jr, Bendixen BH, Kappelle LJ, Biller J, Love BB, Gordon DL, Marsh EE 3rd: Classification of subtype of acute ischemic stroke. Definitions for use in a multicenter clinical trial. TOAST. Trial of Org 10172 in Acute Stroke Treatment. Stroke. 1993, 24:35-41. 10.1161/01.str.24.1.35

3. Güneş M: Relationship between blood pressure levels during thrombolytic therapy and functional outcomes in patients with middle cerebral artery infarction. J Surg Med. 2020, 4:378-382. 10.28982/josam.718969

4. Weimar C, König IR, Kraywinkel K, Ziegler A, Diener HC; German Stroke Study Collaboration: Age and National Institutes of Health Stroke Scale Score within 6 hours after onset are accurate predictors of outcome after cerebral ischemia: development and external validation of prognostic models. Stroke. 2004, 35:158-162. 10.1161/01.STR.0000106761.94985.8B

5. Fan L, Gui L, Chai EQ, Wei CJ: Routine hematological parameters are associated with short- and long-term prognosis of patients with ischemic stroke. J Clin Lab Anal. 2018, 32:e22244. 10.1002/jcla.22244

6. Wang HY, Shi WR, Yi X, Zhou YP, Wang ZQ, Sun YX: Assessing the performance of monocyte to highdensity lipoprotein ratio for predicting ischemic stroke: insights from a population-based Chinese cohort. Lipids Health Dis. 2019, 18:127. 10.1186/s12944-019-1076-6

7. Kocatürk M, Kocatürk Ö: Assessment of relationship between C-reactive protein to albumin ratio and 90day mortality in patients with acute ischaemic stroke. Neurol Neurochir Pol. 2019, 53:205-211. 10.5603/PJNNS.a2019.0020

8. Güneş M: The correlation of routine hematological parameters with in-hospital mortality and length of hospital stay in patients with large middle cerebral artery infarction. Cureus. 2020, 12:e7886. 10.7759/cureus.7886

9. Güneș M: Is neutrophil/eosinophil ratio at admission a prognostic marker for in-hospital mortality of acute ischemic stroke?. J Stroke Cerebrovasc Dis. 2020, 29:104999. 10.1016/j.jstrokecerebrovasdis.2020.104999

10. Willey JZ, Xu Q, Boden-Albala B, Paik MC, Moon YP, Sacco RL, Elkind MS: Lipid profile components and risk of ischemic stroke: the Northern Manhattan Study (NOMAS). Arch Neurol. 2009, 66:1400-1406. 10.1001/archneurol.2009.210

11. Boehme AK, Esenwa C, Elkind MS: Stroke risk factors, genetics, and prevention . Circ Res. 2017, 120:472-495. 10.1161/CIRCRESAHA.116.308398

12. Miyamoto N, Tanaka Y, Ueno Y, et al.: Demographic, clinical, and radiologic predictors of neurologic deterioration in patients with acute ischemic stroke. J Stroke Cerebrovasc Dis. 2013, 22:205-210. 10.1016/j.jstrokecerebrovasdis.2011.07.018 


\section{Cureus}

13. Powers WJ, Rabinstein AA, Ackerson T, et al.: 2018 guidelines for the early management of patients with acute ischemic stroke: a guideline for healthcare professionals from the American Heart Association/American Stroke Association. Stroke. 2018, 49:e46-e110. 10.1161/STR.0000000000000158

14. Forthofer RN, Lee ES, Hernandez M: Biostatistics: A Guide to Design, Analysis and Discovery . Academic Press, Cambridge, MA; 2007. 10.1016/C2009-0-03861-6

15. Hallenbeck JM: Significance of the inflammatory response in brain ischemia . Acta Neurochir Suppl. 1996, 66:27-31. 10.1007/978-3-7091-9465-2_5

16. Wang Q, Tang XN, Yenari MA: The inflammatory response in stroke . J Neuroimmunol. 2007, 184:53-68. 10.1016/j.jneuroim.2006.11.014

17. Jayaraj RL, Azimullah S, Beiram R, Jalal FY, Rosenberg GA: Neuroinflammation: friend and foe for ischemic stroke. J Neuroinflammation. 2019, 16:142. 10.1186/s12974-019-1516-2

18. Jin R, Yang G, Li G: Inflammatory mechanisms in ischemic stroke: role of inflammatory cells . J Leukoc Biol. 2010, 87:779-789. 10.1189/jlb.1109766

19. Tokgoz S, Kayrak M, Akpinar Z, Seyithanoğlu A, Güney F, Yürüten B: Neutrophil lymphocyte ratio as a predictor of stroke. J Stroke Cerebrovasc Dis. 2013, 22:1169-1174

10.1016/j.jstrokecerebrovasdis.2013.01.011

20. Xing Y, An Z, Yu N, Zhao W, Ning X, Wang J: Low density lipoprotein cholesterol and the outcome of acute ischemic stroke: results of a large hospital-based study. Eur Neurol. 2016, 76:195-201. 10.1159/000450604

21. Lackland DT, Roccella EJ, Deutsch AF, et al.: Factors influencing the decline in stroke mortality: a statement from the American Heart Association/American Stroke Association. Stroke. 2014, 45:315-353. 10.1161/01.str.0000437068.30550.cf 